\title{
Risque de cancer à proximité d'installations nucléaires : études épidémiologiques
}

\author{
D. HUBERT ${ }^{1}$
}

(Manuscrit reçu le 4 avril 2002, accepté le 29 septembre 2002)

RÉSUMÉ Depuis l'identification, en 1983, d'un nombre anormalement élevé de leucémies chez les enfants vivant à proximité de l'usine de retraitement de déchets nucléaires de Sellafield, au Royaume-Uni, de nombreuses études épidémiologiques ont porté sur le risque de cancer ou de leucémie dans les populations voisines des installations nucléaires. Un excès de leucémies de l'enfant a été confirmé au voisinage de Sellafield et mis en évidence autour de quelques autres sites, en particulier Dounreay en Écosse et Krümmel en Allemagne, alors que de nombreuses autres études locales sont restées négatives. Parallèlement, des études portant sur plusieurs sites (au RoyaumeUni, en France, aux États-Unis, en Allemagne, au Canada, au Japon, en Suède, en Espagne) n'ont pas détecté d'excès global de leucémie ou de cancer de l'enfant à proximité des installations nucléaires. De plus, des agrégats de leucémie ont été observés dans des zones éloignées de tout site nucléaire. Des études analytiques ont recherché une cause aux excès de leucémies localisés autour de certains sites. Elles ont permis de rejeter I'hypothèse d'une exposition radioactive de la population. L'hypothèse génétique d'un rôle de l'irradiation des pères avant la conception de leur enfant a été évoquée pour expliquer les excès de leucémies constatés à Sellafield, mais elle n'a pas été confirmée par des études concernant d'autres régions ou d'autres pays. Il semble plus probable que ces excès de leucémies soient en rapport avec des brassages de populations.

ABSTRACT Risk of cancer in the vicinity of nuclear installations: epidemiological studies.

After the observation in $\mathbf{1 9 8 3}$ of a leukaemia cluster among children living near the Sellafield nuclear waste reprocessing plant (United Kingdom), numerous epidemiological studies have been conducted on the risk of cancer or leukaemia in populations in the vicinity of nuclear installations. The leukaemia cluster at Sellafield has been confirmed and some other leukaemia clusters have been identified near a few other nuclear sites, such as Dounreay in Scotland and Krümmel in Germany, but many other local studies were negative. At the same time, studies considering several sites (United Kingdom, France, USA, Germany, Canada, Japan, Sweden, Spain) have not detected any global excess of leukaemia or cancer in children living near nuclear installations. Moreover, leukaemia clusters have been observed in areas far from any nuclear site. Analytic studies have looked for a cause explaining the leukaemia clusters located near some nuclear sites. They could reject the hypothesis of a radioactive exposure of the population. The genetic hypothesis of paternal preconceptional irradiation has been suggested to explain the leukaemia cluster at Sellafield, but it has not been confirmed by studies from other areas or other countries. These leukaemia clusters seem more likely to be related to population mixing.

1 F́lectricité de France, Service de Radioprotection, 22-28 rue Joubert, 75009 Paris, France. 


\section{Introduction}

En novembre 1983, une émission de télévision anglaise a signalé dans le village de Seascale, proche du centre de retraitement de Sellafield, un nombre anormalement élevé de leucémies chez les enfants de moins de 10 ans (5 leucémies observées pour 0,45 attendue). À la suite de cette émission, un groupe de travail réuni par le gouvernement anglais a confirmé l'existence de cet excès de leucémies dans la population âgée de moins de 25 ans résidant autour de Sellafield ( 7 cas pour moins de 1 attendu; Black, 1984).

Des études se sont alors poursuivies, non seulement au Royaume-Uni, mais aussi dans de nombreux autres pays, y compris la France. On distingue des études descriptives et des études analytiques (Hubert, 1991 ; Hubert et Hill, 1994 ; Laurier, 1999).

Les études descriptives, réalisées autour d'un site ou de plusieurs sites, ont pour but de confirmer ou d'infirmer une augmentation de leucémies ou d'autres cancers à proximité de sites nucléaires. Il s'agit alors d'études où l'on compare le nombre de cas ou de décès de la population résidant autour des sites nucléaires, appelée «population exposée ", au nombre de cas ou de décès « attendus » dans une population de référence (population nationale ou régionale).

Dans l'éventualité d'études positives, sont réalisées des études analytiques cas-témoins à la recherche de facteurs de risque associés aux excès de cancers. On compare alors l'exposition de malades à celle de personnes témoins non malades.

\section{2. Études descriptives locales}

Ces études autour d'un site nucléaire donné se sont développées dès 1984, d'abord au Royaume-Uni, puis dans de nombreux autres pays (Tabs. I et II).

\subsection{Royaume-Uni}

À la suite du rapport Black (1984), plusieurs autres études ont confirmé l'excès de leucémies chez les jeunes de 0 à 24 ans vivant autour de Sellafield (Draper et al., 1993 ; COMARE, 1996). Cet excès était limité aux jeunes nés à Seascale et ne concernait pas les enfants scolarisés dans le village de Seascale mais nés ailleurs (Gardner et al., 1987). L'excès de leucémies et lymphomes non-hodgkiniens chez les jeunes de 0 à 24 ans vivant au voisinage de Sellafield persistait dans l'étude réalisée en 1996 par le COMARE (Comittee on Medical Aspects of Radiation in the Environment) : 8 cas de 1963 à 1992 pour 0,65 attendu, alors qu'il n'y a pas d'excès statistiquement significatif pour les autres cancers. 
RISQUE DE CANCER À PROXIMITÉ D'TNSTALLATIONS NUCLÉAIRES

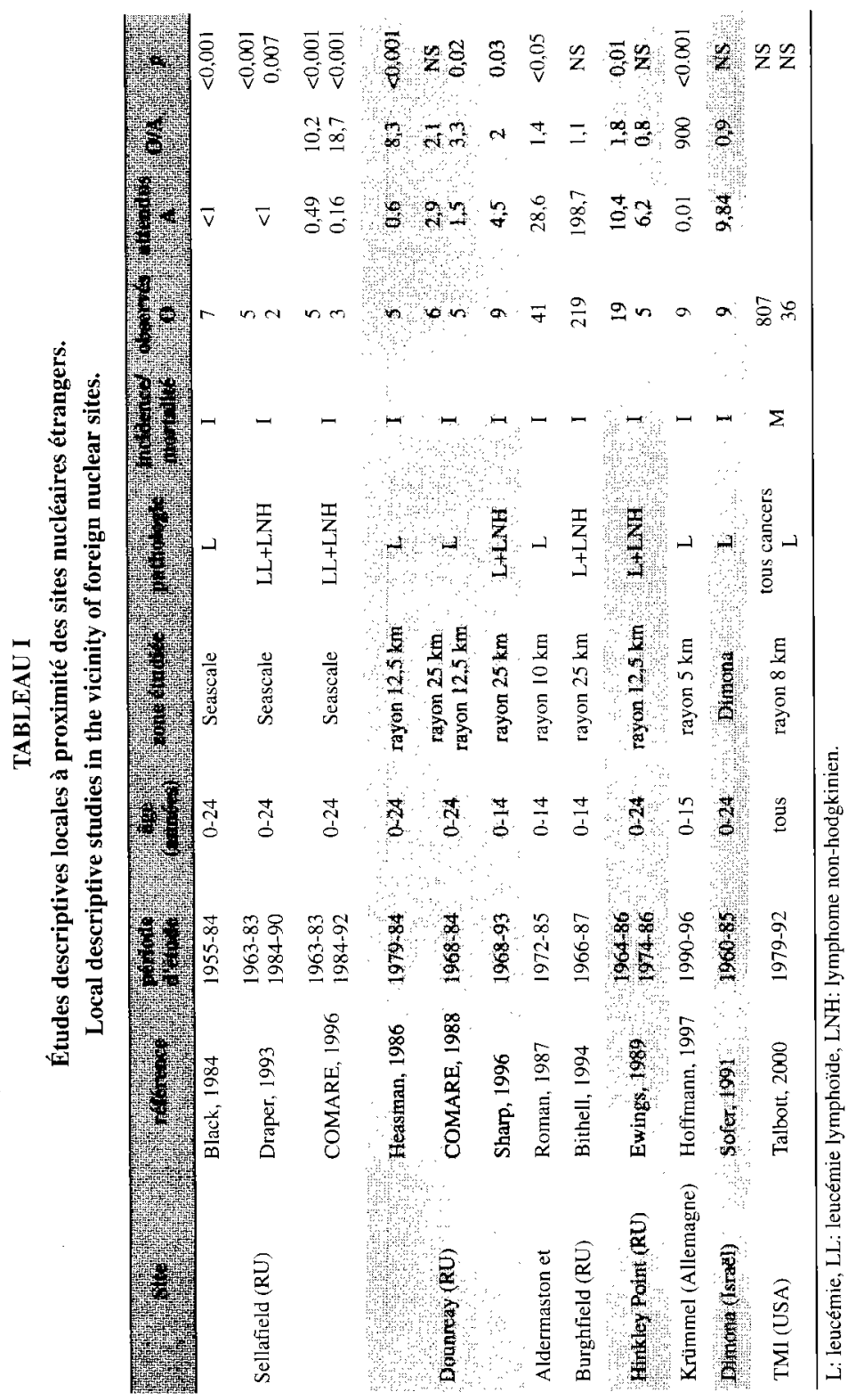


D. HUBERT

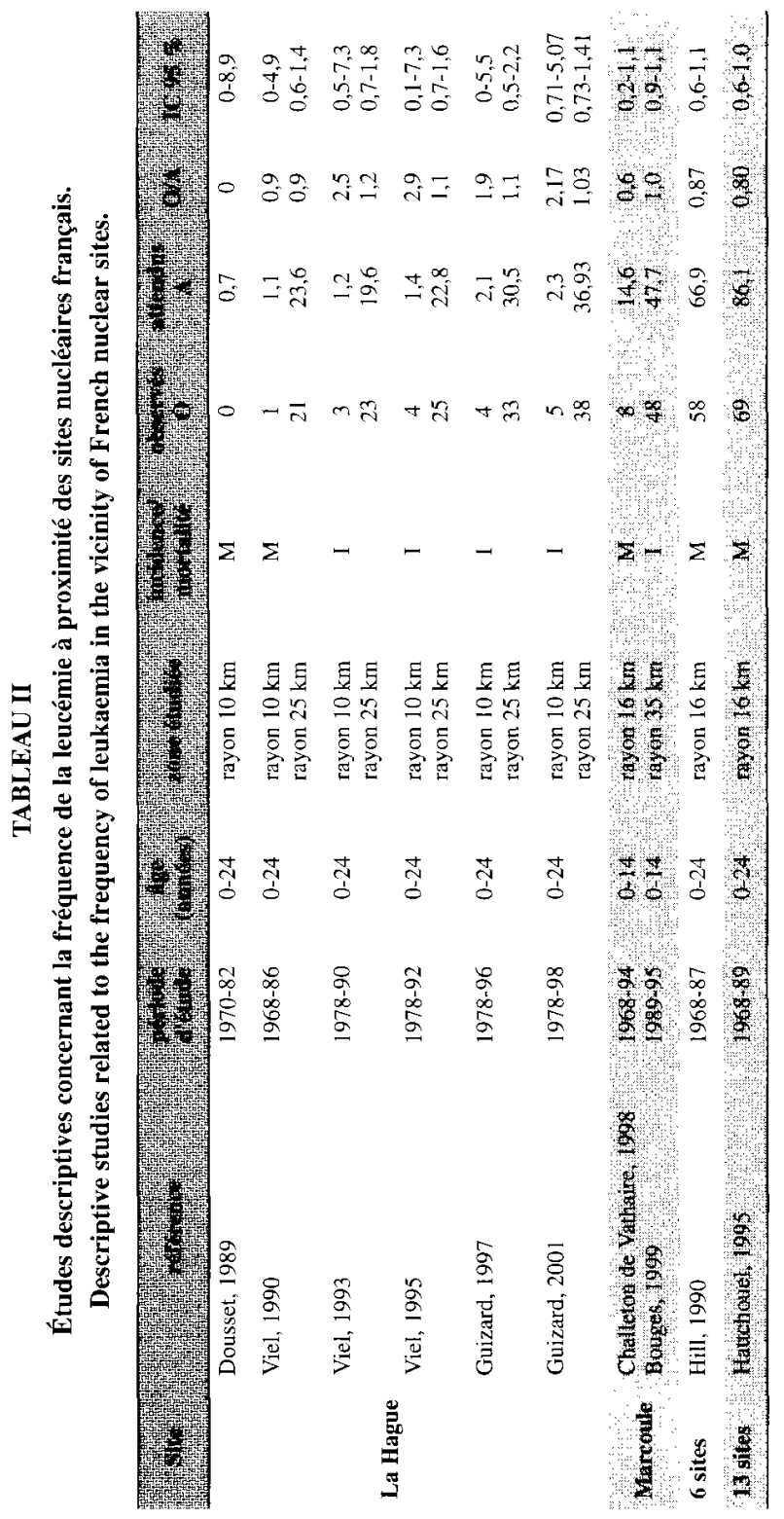


D'autres études, réalisées à partir de 1986 autour de l'usine de retraitement de Dounreay, en Écosse, ont également mis en évidence un excès statistiquement significatif de leucémies chez les jeunes de la même tranche d'âge ( 5 cas observés pour 0,6 attendu) (COMARE, 1988 ; Heasman et al., 1986). Ces cas de leucémie se concentraient sur la période la plus récente (1979-84) et dans la zone la plus proche de l'installation (de $12,5 \mathrm{~km}$ de rayon). Contrairement à Sellafield, cet excès n'était pas limité aux enfants nés dans la région (Black et al., 1992). En revanche, il persiste dans la période la plus récente ( 9 cas pour 4,5 attendus chez les jeunes de 0 à 14 ans entre 1968 et 1993) (Sharp et al., 1996).

D'autres excès de leucémies ont été rapportés autour d'autres sites nucléaires anglais: l'augmentation de leucémies dans la tranche d'âge 0-14 ans autour d'Aldermaston et Burghfield (Roman et al., 1987) n'a pas été confirmée dans une étude ultérieure (Bithell et al., 1994). De même, à Hinkley Point, l'excès de leucémies ne concerne que la période totale de l'étude de 1964 à 1986, mais non les 10 dernières années étudiées (Ewings et al., 1989).

\section{2. États-Unis}

Plusieurs études publiées entre 1985 et 1990 ont donné des résultats négatifs ou ont trouvé des agrégats non confirmés ultérieurement. Citons en particulier celles sur l'usine d'armements nucléaires de Rocky Flats au Colorado, les centrales nucléaires de San Onofre en Californie, de Pilgrim dans le Massachussets et les centres nucléaires de Hanford et Oak Ridge (Hubert, 1991 ; Laurier, 1999).

Une étude de mortalité a porté sur 32135 habitants de la région de la centrale de Three Mile Island (TMI) à la suite de l'accident de 1979 avec très faibles rejets radioactifs dans l'environnement (Talbott et al., 2000). En comparaison à la population de 3 comtés voisins de Pennsylvanie, la mortalité générale de la population voisine de TMI était augmentée (taux standardisé de mortalité ou SMR à $109 \mathrm{chez}$ les hommes et $118 \mathrm{chez}$ les femmes) en raison d'augmentation des décès par maladie cardiovasculaire ou respiratoire non maligne. Il n'y avait pas d'augmentation des décès par cancer ou leucémie.

\subsection{France}

En raison des excès de leucémies rapportés dans la région d'usines de retraitement de combustible irradié en Angleterre et en Écosse, les études françaises se sont d'abord intéressées au site de La Hague dans le Nord-Cotentin. Les premières études de Dousset (1989) et Viel et Richardson (1990) sont des études de mortalité 
qui n'ont détecté aucun excès de leucémie ni de cancer autre, en particulier chez les jeunes de 0 à 24 ans.

Ultérieurement, des études d'incidence ont été réalisées par Viel pour la période 1978-90 (Viel et al., 1993), puis 1978-92 (Viel et al., 1995) : dans la zone des $35 \mathrm{~km}$ de l'usine, il n'y a pas d'excès significatif. Dans la zone des $10 \mathrm{~km}$, 3 cas ont été observés entre 1978 et 1990 et 1 cas supplémentaire est survenu dans les 2 années suivantes, soient 4 cas observés dans la période totale pour 1,4 attendu ; ceci n'est pas statistiquement significatif $(p=0,06)$ mais approche la limite de significativité. Une étude ultérieure, poursuivie jusqu'en 1996, n'a pas vu apparaître de cas supplémentaire de leucémie entre 0 et 24 ans dans la zone proche (Guizard et al., 1997). Au total, sur la période totale étudiée (1978-96), 33 leucémies ont été répertoriées dans la zone des $35 \mathrm{~km}$ (pour 30,5 attendues) et 4 dans la zone des $10 \mathrm{~km}$ (pour 2,1 attendues).

La mise à jour la plus récente de l'incidence de la leucémie de l'enfant autour de l'usine de La Hague a pris en compte la période 1978-98 (Guizard et al., 2001). Au total, les 38 leucémies répertoriées, chez les jeunes de 0 à 24 ans vivant dans un trayon de $35 \mathrm{~km}$ de La Hague, sont conformes au taux attendu (taux standardisé d'incidence ou SIR 1,03 avec intervalle de confiance (IC) à $95 \% 0,73$ à 1,41). En revanche, 5 cas sont survenus dans le canton de Beaumont-Hague (rayon de $10 \mathrm{~km}$ ) avec un SIR à 2,17, élevé mais non statistiquement significatif (IC $95 \%$ : 0,71-5,07). L'excès devient significatif dans cette zone pour la tranche d'âge 5-9 ans : 3 cas, SIR à 6,38 (IC 95\%:1,32-18,65).

D'autres études réalisées autour du site de Marcoule sont restées négatives :

- d'une part pour les études de mortalité par leucémie et cancer, de 1968 à 1994, dans la zone des $16 \mathrm{~km}$, pour les enfants de moins de 15 ans et pour la population dans son ensemble (Challeton-de Vathaire et al., 1998);

- d'autre part pour une étude d'incidence de la leucémie, de 1985 à 1995, chez les enfants de moins de 15 ans habitant dans un rayon de 35 kilomètres, avec un total de 48 leucémies et 15 lymphomes (Bouges et al., 1999). Dans cette même zone, un seul cancer papillaire thyroïdien a été mis en évidence dans la même période.

Citons également une étude sur l'incidence du cancer différencié de la thyroïde autour de la centrale nucléaire de Chooz (Recacewicz et al., 1993) : de 1979 à 1991, 77 cas de cancer différencié de la thyroïde ont été diagnostiqués chez les habitants dans une zone de $40 \mathrm{~km}$ autour de la centrale, sans augmentation par rapport à la population témoin. Il n'y avait pas non plus d'augmentation de risque chez les plus jeunes ni chez les personnes habitant dans la zone la plus proche de la centrale. 


\subsection{Allemagne}

Un agrégat de leucémies de l'enfant de 0 à 15 ans a été mis en évidence dans une zone de $5 \mathrm{~km}$ autour de la centrale de Krümmel, avec 9 cas observés (pour 0,01 attendu) entre 1990 et 1996 (Hoffmann et al., 1997).

\subsection{Israël}

Une étude a été menée dans la région du Neguev où est implantée la centrale nucléaire de Dimona, concluant à l'absence d'excès de leucémie chez les jeunes de 0 à 24 ans (Sofer et al., 1991). Pour la population des Juifs de moins de 25 ans, le taux d'incidence de la leucémie était de 3,51/100 000 dans la région Ouest du Neguev située à distance de la centrale et de 2,76/100 000 dans la région Est du Neguev plus proche de la centrale.

\section{3. Études descriptives autour de plusieurs sites nucléaires (Tabs. II et III)}

Des études portant sur plusieurs sites d'une même région ou d'un même pays ont été entreprises simultanément aux études ne concernant qu'un seul site. Elles portent alors sur un nombre de cas beaucoup plus élevé et permettent d'accroître la puissance statistique.

Là encore, ces études ont débuté au Royaume-Uni. Une étude de mortalité qui a porté sur 15 sites d'Angleterre et du Pays de Galles (en 1989) (Cook-Mozaffari et al., 1989b) a trouvé un excès de $15 \%$ de leucémie des jeunes de moins de 25 ans à proximité des sites nucléaires, sans lien avec la distance au site. Ultérieurement, une étude d'incidence, élargie à 23 sites (Bithell et al., 1994) n'a pas retrouvé d'excès global de leucémies. En revanche, l'excès existe pour les sites de Sellafield et de Burghfield, déjà identifiés dans les études spécifiques à ces sites. L'étude de Sharp et al. (1996), portant sur 7 sites nucléaires écossais, n'a pas mis en évidence d'augmentation globale de leucémie, mais là encore a retrouvé l'agrégat connu de Dounreay.

En France, la première étude de mortalité à proximité de 6 sites nucléaires, en activité avant 1975 , n'a mis en évidence aucun excès de leucémie (58 décès par leucémie observés pour 66,9 attendus), ni de tout autre cancer, dans la population de moins de 25 ans (Hill et Laplanche, 1990). Cette étude, élargie en 1995 à l'ensemble des 13 sites nucléaires en activité avant 1985, est restée négative (Hattchouel et al., 1995, 1996) : 69 décès par leucémie pour 86 attendus dans la classe d'âge 0-24 ans, pas d'augmentation des autres types de cancer dans cette 
D. HUBERT

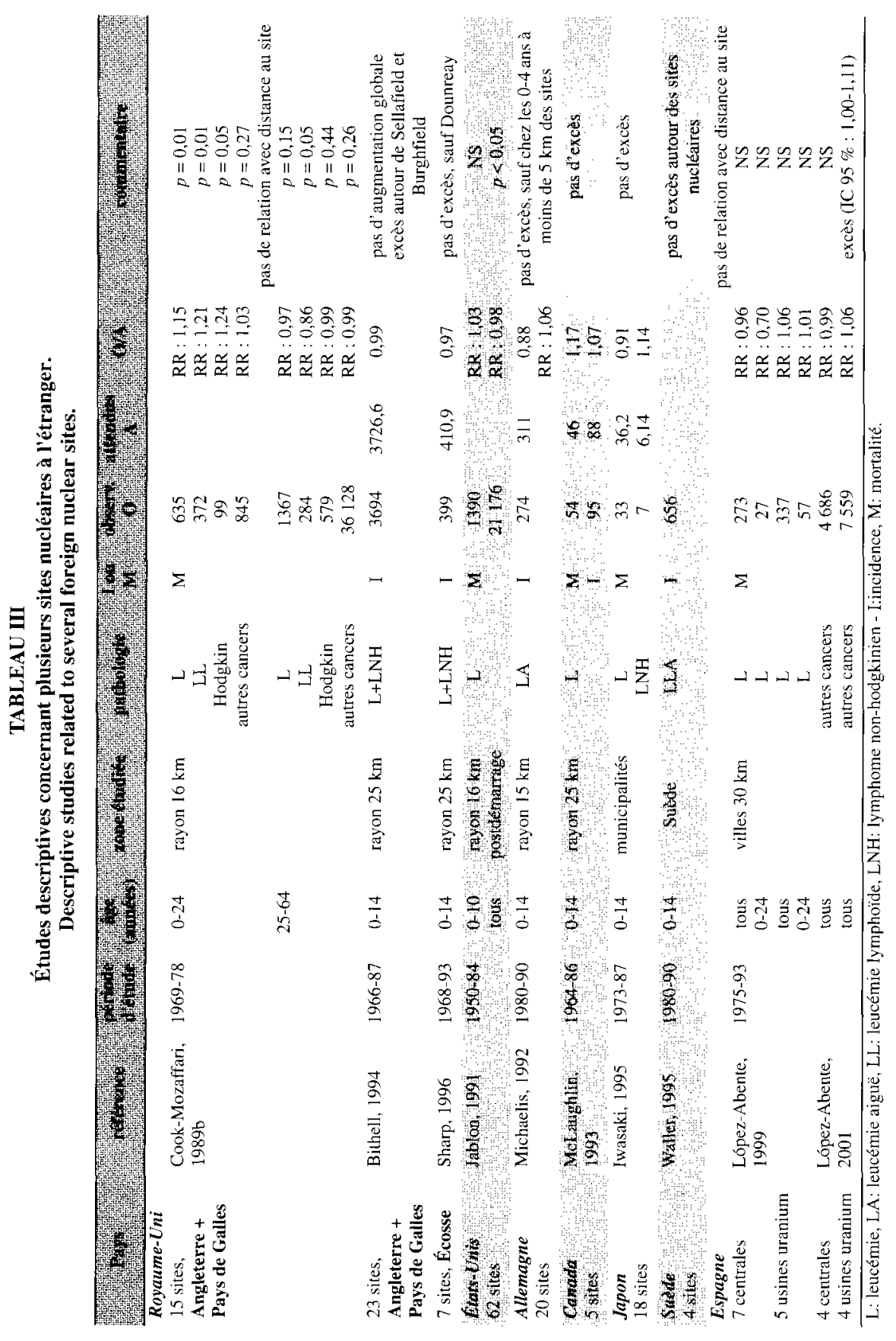


classe d'âge et chez les moins de 65 ans. Il n'y a pas non plus de quelconque relation avec la distance au site nucléaire.

Aux États-Unis, l'étude de Jablon et al. (1991) a pris en compte 62 sites nucléaires et 37200 décès par leucémie ; il existe une augmentation des leucémies de l'enfant dans les régions exposées, mais uniquement avant le démarrage de l'installation, ce qui permet d'exclure un effet des rayonnements ionisants. Après le démarrage de l'installation, on observe un risque de leucémies significativement plus bas autour des installations nucléaires que dans certaines régions témoins.

En Allemagne, Michaelis et al. (1992) ont étudié l'incidence des leucémies aiguës autour de 20 sites. Il n'y a pas d'augmentation pour la population d'enfants de 0 à 14 ans. En revanche, il existe un excès chez les enfants de 0 à 4 ans par rapport aux régions témoins où l'incidence des leucémies dans cette tranche d'âge est anormalement basse (taux standardisé d'incidence ou SIR $=0,75$ ), alors qu'il n'y a pas d'excès par rapport aux taux nationaux $(\mathrm{SIR}=0,96)$.

Au Canada, une étude qui a porté sur 95 cas de leucémies entre 0 et 14 ans, autour de 5 sites nucléaires, est négative (McLaughlin et al., 1993).

Au Japon, aucun excès de leucémie ni de lymphome n'est observé dans la même tranche d'âge, autour de 18 sites nucléaires (Iwasaki et al., 1995).

Une étude suédoise, reprenant, à partir d'un registre national, 656 cas de leucémie lymphoïde aiguë entre 1980 et 1990, n'a trouvé aucune concentration anormale autour des 4 sites nucléaires (Waller et al., 1995).

En Espagne, des études épidémiologiques ont porté sur le risque de décès par leucémie (Lopez-Abente et al., 1999) ou par tumeurs solides (Lopez-Abente et al., 2001) dans la population voisine de centrales nucléaires et d'usines de fabrication de combustible pour la période 1975-93.

Pour l'ensemble des sites nucléaires étudiés, il n'apparaît pas d'excès de leucémie ou autre cancer hématologique dans la population avoisinante ; en particulier, il n'y a pas d'excès de leucémie chez les moins de 25 ans (LopezAbente et al., 1999).

L'étude de la mortalité par cancer solide ne trouve pas d'excès à proximité des centrales nucléaires considérées dans leur ensemble. En revanche, on observe une augmentation des cancers en général dans les villes voisines des usines de fabrication de combustible nucléaire (RR : 1,06; IC 95\%:1,00-1,11), attribuée à une augmentation du cancer broncho-pulmonaire (RR : 1,12 - IC $95 \%: 1,01-1,24$ ) et du cancer du rein (RR : 1,37; IC 95\%: 1,07-1,76) (Lopez-Abente et al., 2001). 
Cependant, le risque de cancer n'est pas lié à la proximité de l'installation nucléaire, et n'est pas différent avant et après le démarrage du site. Pour expliquer l'excès de cancers autour des usines de fabrication de combustible nucléaire, les auteurs avancent l'hypothèse d'un impact environnemental lié aux mines d'uranium ou d'autres minerais, nombreuses à proximité des usines de fabrication de combustible nucléaire, ou à une exposition au radon.

L'analyse de chaque site en particulier fait apparâtre quelques résultats positifs pour certains types de cancers autour de certains sites, dont l'interprétation reste difficile en raison du faible nombre de cas: (1) pour les cancers hématologiques, excès de leucémies dans la population voisine de l'usine de fabrication de combustible nucléaire de Andújar (RR : 1,30 avec IC 95\%:1,02$1,64)$; excès de myélome dans la population voisine de la centrale nucléaire de Zorita (RR : 4,35 avec IC $95 \%$ : 1,50-12,64) (Lopez-Abente et al., 1999) ; (2) pour les tumeurs solides, augmentation du cancer de l'estomac autour de Garoña, du cancer du rein et du cancer des os dans la zone des $15 \mathrm{~km}$ autour de Vandellós, et de tumeurs du tissu conjonctif autour de Zorita et Almaraz (Lopez-Abente et al., 2001).

\section{4. Études descriptives concernant des sites potentiels}

Il est intéressant de savoir que certaines études se sont intéressées au risque de leucémies dans des régions n'ayant pas de site nucléaire, mais où la construction d'une centrale avait été envisagée. Ainsi, sur 8 «sites potentiels » d'Angleterre et du Pays de Galles, Cook-Mozaffari et al. (1989a) ont trouvé un excès de leucémie et de maladie de Hodgkin dans la population 0-24 ans. Ce résultat n'a pas été confirmé dans l'étude ultérieure de Bithell et al. (1994).

En Allemagne, Michaelis et al. (1992), considérant 6 sites potentiels, ont montré une augmentation des leucémies chez les jeunes de moins de 15 ans.

\section{5. Études analytiques}

Plusieurs hypothèses ont été soulevées pour essayer d'expliquer les rares excès de leucémies localisés dans certaines régions, en particulier une exposition radioactive ou d'autres facteurs environnementaux, un effet génétique ou une transmission infectieuse.

\subsection{Exposition radioactive et facteurs environnementaux}

Divers rapports anglais ont rejeté comme cause possible la seule exposition aux différentes sources d'irradiation. Ainsi, pour le cas de Sellafield, en additionnant 
les diverses sources d'exposition aux rayonnements que sont la radioactivité naturelle, l'exposition médicale, les retombées d'essais atomiques et de Tchernobyl ainsi que les rejets de l'usine de Sellafield, en fonction des risques connus des rayonnements ionisants, on prévoyait 0,46 cas de leucémie dans la période 1955-92, alors qu'il y en a eu 12 (COMARE, 1996).

Une étude française cas-témoins réalisée autour de La Hague a suggéré le rôle de la fréquentation des plages locales, de la consommation de poissons et fruits de mer locaux, ainsi que la durée de résidence dans des maisons en granit (Pobel et Viel, 1997). Cette étude a été largement critiquée, principalement par des épidémiologistes (petite taille de l'échantillon, biais potentiels liés à un interrogatoire rétrospectif, ... ; Clavel et Hemon, 1997 ; Law et Roman, 1997 ; Wakeford, 1997).

Suite à ces différentes polémiques, une étude radioécologique a été réalisée en 1997 par le Groupe radioécologie Nord-Cotentin (regroupant des représentants d'organismes de radioprotection français, d'exploitants nucléaires, d'associations environnementales et des experts étrangers), afin d'évaluer de façon réaliste l'exposition aux rayonnements ionisants de la population 0-24 ans résidant à proximité de l'usine de retraitement de La Hague (Laurier et al., 2000 ; Rommens et al., 2000). La cohorte reconstituée comporte 6656 individus nés entre 1954 et 1996 ayant résidé au moins un an dans le canton de Beaumont-Hague entre 1978 et 1996 avant l'âge de 25 ans. Sur la base de l'estimation des doses reçues, le nombre de cas de leucémie radio-induite au sein de cette population, attribuable à l'exposition due aux rejets des installations nucléaires locales, est inférieur à 0,002 sur la période 1978-96, ce qui est faible en regard des 4 cas de leucémie observés sur la même période.

Une étude cytogénétique a été réalisée en Allemagne chez 42 enfants de la région d'Elbmarsch, proche de la centrale nucléaire de Krümmel où a été décrit un cluster de leucémies de l'enfant (Bruske-Hohlfeld et al., 2001), dans le but de rechercher un indicateur d'exposition élevée des enfants durant leur enfance. Les aberrations chromosomiques à type de dicentriques et d'anneaux n'étaient pas plus fréquentes que dans une population témoin de 30 enfants.

\subsection{Hypothèse génétique}

Dans l'étude cas-témoins publiée par Gardner en 1990 (Gardner et al., 1990), portant sur des jeunes de la région de Sellafield, une proportion plus élevée de cas que de témoins avaient un père employé dans l'industrie nucléaire, mais la différence n'était pas significative. En revanche, 4 pères de 46 enfants leucémiques avaient reçu une dose cumulée avant la conception supérieure à $100 \mathrm{mSv}$ contre 


\section{HUBERT}

seulement 3 des 276 pères d'enfants témoins, ce qui correspond à un risque de leucémie 8,4 fois plus élevé pour les enfants dont le père avait reçu une dose supérieure à $100 \mathrm{mSv}$. Les mêmes constatations étaient faites lorsque l'exposition du père dépassait une dose de $10 \mathrm{mSv}$ dans les 6 mois avant la conception.

Plusieurs autres études cas-témoins ont eu pour but d'explorer cette hypothèse de Gardner, au Royaume-Uni et au Canada : elles ont toutes été négatives (Urquhart et al., 1991 ; McLaughlin et al., 1992 ; Roman et al., 1993).

En 1997, une enquête cas-témoins de grande ampleur réalisée au Royaume-Uni à partir du Registre national des leucémies et du Registre des travailleurs du nucléaire, a conclu que les pères d'enfants leucémiques avaient plus souvent travaillé dans l'industrie nucléaire (risque relatif $\mathrm{RR}=1,77$ ), mais sans que l'on retrouve de relation dose-effet (Draper et al., 1997). En particulier, le risque était particulièrement faible pour les enfants dont le père avait reçu une dose totale de $100 \mathrm{mSv}$ ou plus avant la conception $(\mathrm{RR}=0,46)$.

De même, une étude de cohorte, réalisée sur 46107 enfants de moins de 25 ans de travailleurs de l'industrie nucléaire du Royaume-Uni, a trouvé une incidence de cancers et de leucémies chez ces enfants comparable à celle de la population générale (Roman et al., 1999). Le taux de leucémie chez les enfants dont le père avait reçu une dose de plus de $100 \mathrm{mSv}$ avant la conception était 5,8 fois plus élevé que celui des enfants dont le père ne travaillait pas dans l'industrie nucléaire avant la conception, mais cette constatation ne repose que sur 3 cas chez les pères exposés (dont 2 déjà inclus dans l'étude initiale de Gardner). Par ailleurs, on n'a pas trouvé de relation dose-effet dans cette étude.

L'étude de l'incidence des leucémies et lymphomes non-hodgkiniens chez les enfants des travailleurs de Sellafield a été reprise par Dickinson et Parker (2002). Par rapport à l'étude de Gardner et al. (1990), il s'agit d'une étude de cohorte étendue à toute la région de Cumbria et rallongée de 6 années (de 1950 à 1991). Treize cas de leucémies et lymphomes non hodgkiniens sont diagnostiqués parmi les enfants des 9859 travailleurs de Sellafield exposés aux rayonnements ionisants avant la conception de leur enfant, contre $197 \mathrm{cas}$ chez les enfants de père non employé à Sellafield; ceci représente un risque accru dans la cohorte des travailleurs exposés (RR $=1,9$ avec IC $95 \%: 1$ à 3,1). Il faut noter que 10 des 13 cas de leucémie chez les enfants des travailleurs étaient déjà inclus dans l'étude de Gardner et al. (1990). On retrouve une relation significative entre le risque de leucémie et la dose d'irradiation externe reçue par le père avant la conception, mais elle n'est pas significative chez les enfants de plus de 7 ans, pour la période la plus récente 1969-91, pour les enfants nés en dehors du village de Seascale et si l'on exclut les cas déjà analysés par Gardner. Elle est moins nette que dans l'étude de Gardner. 
En revanche, chez les enfants des travailleurs de Sellafield, il n'apparaît pas de relation entre le risque de tumeur solide et la dose d'irradiation externe reçue par leur père avant leur conception (Dickinson et al., 2002b).

\subsection{Hypothèse infectieuse}

Kinlen et al. (1990) émettent l'hypothèse que la leucémie de l'enfant est une réaction rare à une infection commune, probablement virale, dont la transmission est facilitée par les brassages de population. Ainsi, ne seraient pas en cause les rayonnements ionisants, mais des brassages de populations, liés à un afflux massif de populations dans une région rurale isolée, au moment de la construction et du démarrage de l'installation. Des mouvements des travailleurs de l'industrie du pétrole, arrivés en Écosse en grand nombre entre 1979 et 1983, pourraient en partie expliquer les excès de leucémie observés autour de Dounreay (Kinlen et al., 1993).

De même, les mouvements de population ont été mis en avant pour expliquer les excès de leucémies aiguës lymphoblastiques et de lymphomes nonhodgkiniens, tout particulièrement chez les jeunes enfants, dans la région de Cumbria où est située la centrale de Sellafield et dans le village voisin de Seascale (Dickinson et Parker, 1999). Les mêmes auteurs ont élargi leur étude en considérant 10194 cas de leucémies et lymphomes non-hodgkiniens enregistrés chez des enfants de moins de 15 ans en Angleterre et au Pays de Galles entre 1966 et 1987 (Dickinson et al., 2002a) ; un risque accru de leucémie persiste pour les régions à taux d'immigration élevée, mais il est moins net que précédemment et n'est évident que dans les zones urbaines. Enfin, Dickinson et Parker (2002) confirment le rôle des facteurs démographiques, en particulier des brassages de population, sur le risque de leucémie chez les enfants des travailleurs de Sellafield nés à Seascale : pour les enfants de moins de 7 ans nés à Seascale entre 1969 et 1991, le RR passe de 15,4 (IC 2,5 à 5) à 4,6 (IC 0,7 à 15) après ajustement pour les facteurs démographiques.

Une étude réalisée dans les îles Orkney et Shetland montre que le nombre de leucémies de l'enfant y a augmenté lors de la seconde guerre mondiale, alors qu'elles connaissaient une augmentation de population par afflux de militaires (Kinlen et Balkwill, 2001).

Une étude est en cours pour explorer cette hypothèse dans la population voisine de La Hague (Guizard et al., 2001).

Cette hypothèse de brassage de population serait susceptible d'expliquer un léger excès de tumeurs solides non statistiquement significatif chez les enfants des travailleurs de Sellafield exposés aux rayonnements ionisants en comparaison aux 
enfants de Cumbria dont le père ne travaillait pas à Sellafield $(\mathrm{RR}=1,5$; IC $95 \%$ : 0,9 à 2,4) (Dickinson et al., 2002b).

\section{Conclusion}

Les études descriptives dont nous disposons actuellement mettent en évidence certains agrégats de leucémie des enfants autour de quelques sites nucléaires, essentiellement autour de Sellafield et Dounreay au Royaume-Uni et de Krümmel en Allemagne. Néanmoins, les études regroupant plusieurs sites ne trouvent pas d'excès global. Les études analytiques n'ont pas apporté de réponse définitive. Il semble que les brassages de population soient en partie responsables de ces excès. L'hypothèse génétique d'un rôle de l'irradiation des pères avant la conception de l'enfant n'est pas démontrée mais ne peut pas être totalement écartée en fonction des résultats de l'étude récente de Dickinson et Parker (2002).

\section{RÉFÉRENCES}

Bithell J.F., Dutton S.J., Draper G.J., Neary N.M. (1994) Distribution of childhood leukaemias and non-hodgkin lymphomas near nuclear installations in England and Wales, BMJ 309, 501-505.

Black D. (1984) Investigation of the possible increased incidence of cancer in West Cumbria, report of the independant advisory group. HMSO, Londres.

Black R., Urquhart J.D., Kendrick S.W., Bunch K.J., Warner J., Jones D.A. (1992) Incidence of leukaemia and other cancers in birth and school cohorts in the Dounreay area, BMJ 304, 14011405.

Bouges S., Daures J., Hebrard M. (1999) Incidence des leucémies aiguës, lymphomes et cancers thyroïdiens chez les enfants de moins de 15 ans vivant autour du site nucléaire de Marcoule de 1985 à 1995, Rev. Épidémiol. Santé Publ. 47, 205-217.

Bruske-Hohlfeld I., Scherb H., Bauchinger M., Schmid E., Fender H., Wolf G., Obe G., SchmitzFeuerhake I., Schroder H., Stephan G., Csicsaky M., Wichmann H.E. (2001) A cluster of chilhood leukemias near two neighbouring nuclear installations in Northern Germany: prevalence of chromosomal aberrations in peripheral blood lymphocytes, Int. J. Radiat. Biol. 77, 111-116.

Challeton-de Vathaire C., de Vathaire F., Bourguignon M. (1998) Étude de la mortalité par cancer entre 1963 et 1994 autour du site nucléaire de Marcoule, Rayonn. Ionis. 27, 41-46.

Clavel J., Hemon D. (1997) Leukaemia near La Hague nuclear Plant. Bias could have been introduced into study, $B M J 314,1553$.

COMARE (1988) Committee on Medical Aspects of Radiation in the Environment, Second report: Investigation of the possible increased incidence of leukaemia in young people near Dounreay nuclear establishment, Caithness, Scotland. HMSO, Londres.

COMARE (1996) Committee on Medical Aspects of Radiation in the Environment, Fourth report: The incidence of cancer and leukaemia in young people in the vicinity of the Sellafield site, West Cumbria. Further studies and an update of the situation since the publication of the Black Advisory group in 1984. HMSO, Londres, $179 \mathrm{p}$.

Cook-Mozaffari P.J., Darby S.C., Doll R. (1989a) Cancer near potential sites of nuclear installations, Lancet 2, 1145-1147. 
Cook-Mozaffari P.J., Darby S.C., Doll R., Forman D., Hermon C., Pike M.C., Vincent T. (1989b) Geographical variations in mortality from leukaemia and other cancers in England and Wales in relation to proximity to nuclear installations, 1969-1978, Brit. J. Cancer 59, 476-485.

Dickinson H.O., Parker L. (1999) Quantifying the effect of population mixing on childhood leukaemia risk: the Seascale cluster, Brit. J. Cancer 81, 144-151.

Dickinson H.O., Parker L. (2002) Leukaemia and non-Hodgkin's lymphoma in children of male Sellafield workers, Int. J. Cancer 99, 437-444.

Dickinson H.O., Hammal D.M., Bithell J.F., Parker L. (2002a) Population mixing and childhood leukaemia and non-Hodgkin's lymphoma in census wards in England and Wales, 1966-87, Brit. J. Cancer 86, 1411-1413.

Dickinson H.O., Parker L., Salotti J., Birch P. (2002b) Paternal preconceptional irradiation, population mixing and solid tumors in the children of radiation workers (England), Cancer Causes Control 13, 183-189.

Dousset M. (1989) Cancer mortality around La Hague nuclear facilities, Health Phys. 56, 875-884.

Draper G.J., Stiller C.A., Cartwright R.A., Craft A.W., Vincent T.J. (1993) Cancer in Cumbria and in the vicinity of the Sellafield nuclear installation, 1963-90, BMJ 306, 89-94.

Draper G.J. et al. (1997) Cancer in the offspring of radiation workers: a record linkage study, BMJ 315, 1181-1188.

Ewings P.D., Bowie C. Philips M.J., Johnson S.A.N. (1989) Incidence of leukaemia in young people in the vicinity of Hinkley Point nuclear power station, 1959-86, BMJ 299, 289-293.

Gardner M.J., Hall A.J., Downes S., Terrell J.D. (1987) Follow-up study of children born to mothers resident in Seascale, West Cumbria (birth cohort), BMJ 295, 822-827.

Gardner M.J., Snee M.P., Hall A.J., Powell C.A., Downes S., Terrell J.D. (1990) Results of a casecontrol study of leukaemia and lymphoma among young people near Sellafield nuclear plant in West Cumbria, BMJ 300, 423-429.

Guizard A.V., Spira A., Troussard X., Collignon A. (1997) ARKM, Incidence des leucémies de 0 à 24 ans dans le Nord-Cotentin, Rev. Épidemiol. Santé Publ. 45, 530-535.

Guizard A.V., Boutou O., Pottier D., Troussard X., Pheby D., Launoy G., Slama R., Spira A. (2001) The incidence of childhood leukaemia around the La Hague nuclear waste reprocessing plant (France): a survey for the years 1978-1998, J. Epidemiol. Comm. Health 55, 469-474.

Hattchouel J.M., Laplanche A., Hill C. (1995) Leukaemia mortality around French nuclear sites, Brit. J. Cancer 71, 651-653.

Hattchouel J.M., Laplanche A., Hill C. (1996) Cancer mortality around French nuclear sites, Ann. Epidemiol. 6, 126-129.

Heasman M.A., Kemp I.W., Urquhart J.D., Black R. ( 1986) Childhood leukaemia in nothern Scotland, Lancet 1, 266.

Hill C., Laplanche A. (1990) Overall mortality and cancer motality around French nuclear sites, Nature 347, $755-757$.

Hoffmann W., Dieckmann H., Schmitz-Feverhake I. (1997) A cluster of childhood leukaemia near a nuclear reactor in Northern Germany, Arch. Environ. Health 52, 275-280.

Hubert D. (1991) Leucémies autour des installations nucléaires anglaises, Radioprotection 26, 351372 .

Hubert D., Hill C. (1994) Étude des populations habitant près des installations nucléaires, Radioprotection 29, 89-99.

Iwasaki T., Nishizawa K., Murata M. (1995) Leukaemia and lymphoma mortality in the vicinity of nuclear power stations in Japan, 1973-1987, J. Radiol. Protect. 15, 27 I-288. 


\section{HUBERT}

Jablon S., Hrubec Z., Boice J.D. (1991) Cancer in populations living near nuclear facilities, a survey of mortality nationwide and incidence in two states, JAMA 265, 1403-1408.

Kinlen L.J., Balkwill A. (2001) Infective cause of chilhood leukemia and wartime population mixing in Orkney and Shetland, UK, Lancet 357, 858.

Kinlen L.J., Clarke K., Hudson C. (1990) Evidence from population mixing in British new towns 194685 of an infective basis for childhood leukaemia, Lancet 336, 577-582.

Kinlen L.J., O’Brien F., Clarke K., Balkwill A., Matthews F. (1993) Rural population mixing and childhood leukaemia: effects of the North Sea oil industry in Scotland, including the area near Dounreay nuclear site, BMJ 306, 743-748.

Laurier D. (1999) Leucémies chez les moins de 25 ans autour d'installations nucléaires en France et à l'étranger : revue des études épidémiologiques descriptives, Radioprotection 34, 149-176.

Laurier D., Rommens C., Drombry-Ringeard C., Merle-Szeremeta A., Degrange J.P. (2000) Évaluation du risque de leucémie radio-induite à proximité d'installations nucléaires : l'étude radioécologique Nord-Cotentin, Rev. Épidemiol. Santé Publ. 2, 24-36.

Law G., Roman E. (1997) Leukaemia near La Hague nuclear Plant. Study design is questionable, $B M J$ 314, 1553.

Lopez-Abente G., Aragones N., Pollan M., Ruiz M., Gandarillas A. (1999) Leukemia, lymphomas, and myeloma mortality in the vicinity of niuclear power plants and nuclear fuel facilities in Spain, Cancer Epidemiol. Biomark. Prev. 8, 925-934.

Lopez-Abente G., Aragones N., Pollan M. (2001) Solid-tumor mortality in the vicinity of uranium cycle facilities and nuclear power plants in Spain, Environ. Health Perspect. 109, 721-729.

McLaughlin J.R., Anderson T.W., Clarke E.A., King W. (1992) Occupational exposure of fathers to ionizing radiaiton and the risk of leukaemia in offspring - a case-control study. Atomic energy control board, Ottawa.

McLaughlin J.R., Clarke E.A., Nishri E.D., Anderson T.W. (1993) Childhood leukaemia in the vicinity of Canadian nuclear facilities, Cancer Causes Control 4, 51-58.

Michaelis J., Keller B., Haaf G., Kaatsch P. (1992) Incidence of childhood malignancies in the vicinity of West German nuclear power plants, Cancer Causes Control 3, 255-263.

Pobel D., Viel J.F. (1997) Case-control study of leukaemia among young people near La Hague nuclear reprocessing plant: the environmental hypothesis revisited, $B M J 314,101-106$.

Recacewicz C., de Vathaire F., Delisle M.J. (1993) Differentiated thyroid carcinoma incidence around the French nuclear power plant in Chooz, Lancet 341, 493.

Roman E., Beral V., Carpenter L., Watson A., Barton C., Ryder H., Aston D.L. (1987) Childhood leukaemia in the West Berkshire and Basingstoke and North Hampshire district health authorities in relation to nuclear establishments in the vicinity, BMJ 294, 597-602.

Roman E., Watson A., Beral V., Buckle S., Bull D., Baker K., Ryder H., Barton C. (1993) Case-control study of leukaemia and non-Hodgkin's lymphoma among children aged 0-4 years living in West Berkshire and North Hampshire health districts, $B M J$ 306, 615-621.

Roman E., Doyle P., Maconochie N., Davies G., Smith P.G., Beral V. (1999) Cancer in children of nuclear industry employees: report on children aged under 25 years from nuclear industry family study, BMJ 318, 1443-1450.

Rommens C., Laurier D., Sugier A. (2000) Methodology and results of the Nord-Cotentin radioecological study, J. Radiol. Prot. 20, 349-351.

Sharp L., Black R.J., Harkness E.F., McKinney P.A. (1996) Incidence of childhood leukaemia and nonhodgkin's lymphoma in the vicinity of nuclear sites in Scotland, 1968-93, Occup. Environ. Med. $\mathbf{5 3}, 823-831$. 
Sofer T., Goldsmith J.R., Nusselder I., Katz L. (1991) Geographical and temporal trends of childhood leukemia in relation to the nuclear plant in the Neguev, Israel, 1960-1985, Pub. Health Rev. 19, 191-198.

Talbott E.O., Youk A.O., McHugh K.P., Shire J.D., Zhang A., Murphy B.P., Engberg R.A. (2000) Mortality among the residents of the Three Mile Island accident area: 1979-1992, Environ. Health Perpect. 108, 545-552.

Urquhart J.D., Black R.J., Muirhead M.J., Sharp L., Maxwell M., Eden O.B., Adams Jones D. (1991) Case-control study of leukaemia and non-Hodgkin's lymphoma in children in Caithness near the Dounreay nuclear installation, $B M J 302,687-692$.

Viel J.F., Richardson S.T. (1990) Childhood leukaemia around the La Hague nuclear waste reprocessing plant, $B M J$ 300, 580-581.

Viel J.F., Richardson S., Danel P., Boutard P., Malet M., Barrelier P., Reman O., Carré A. (1993) Childhood leukaemia incidence in the vicinity of La Hague nuclear waste reprocessing facility, Cancer Causes Control 4, 341-343.

Viel J.F., Pobel D., Carré A. (1995) Incidence of leukaemia in young people around the La Hague nuclear waste reprocessing plant: a sensitivity analysis, Stat. Med. 14, 2459-2472.

Wakeford R. (1997) Leukaemia near La Hague nuclear Plant. Scientific context is needed, BMJ 314, 1553-1554.

Waller L.A., Turnvull B.W., Gustafsson G., Hjalmars U., Andersson B. (1995) Dectection and assessment of clusters of disease : an application to nuclear power plant facilities and childhood leukaemia in Sweden, Stat. Med. 14, 3-16. 